\title{
Infrastructure Management Process Maturity Model: Development and Testing
}

\author{
Jehan Zeb ${ }^{1}$, Thomas Froese ${ }^{1} \&$ Dana Vanier ${ }^{1}$ \\ ${ }^{1}$ Department of Civil Engineering, The University of British Columbia, Vancouver, Canada \\ Correspondence: Jehan Zeb, Department of Civil Engineering, The University of British Columbia, Vancouver, \\ British Columbia, V6T 1Z4, Canada. Tel: 1-604-822-2739. E-mail: jzmir1@interchange.ubc.ca
}

\author{
Received: June 6, 2013 Accepted: September 16, 2013 Online Published: September 25, 2013 \\ doi:10.5539/jsd.v6n11p1 URL: http://dx.doi.org/10.5539/jsd.v6n11p1
}

\begin{abstract}
To better serve society, infrastructure organizations must manage their civil infrastructure systems effectively and efficiently, employing best practices in infrastructure management and relevant information systems. As information systems mature, communications follow a general trend away from informal human-to-human communications towards computer-to-computer information exchange. For efficient implementation of computer-based exchange of information, these communications must be formally described. As part of a larger study into the formalization of communications within the infrastructure industry, this paper examines the level to which work processes and communications are formalized and designed at present within the domain of infrastructure management. The research adopts a maturity model approach. There are many maturity models available in different industries, but their focus is on the maturity of the way work processes and communication are operated and managed, not the way these work processes and communications are formalized and designed. To address the issue, an Infrastructure Management-Process Maturity Model (IM-PMM) is developed to assess the degree to which work processes and communications are formalized within a specific engineering domain, namely infrastructure management. A five-step approach is used to develop the IM-PMM: define the problem, compare existing maturity models, develop the model, apply the model, and evaluate the maturity model.

This paper describes the development and application of the Infrastructure Management-Process Maturity Model (IM-PMM) that can benchmark the current level of maturity of work processes and communications in the domain of infrastructure management. The proposed IM-PMM uses a scale of five levels of maturity (stages) and uses three core elements (i.e. process/transaction map definition, actor/role definition, and information definition) to benchmark existing work processes, plus one additional element (message definition) to benchmark existing communications. The proposed model has been applied and tested in the domain of infrastructure management using a structured interview approach. The resulting data show that existing work processes and communications are typically accomplished in an $a d$ hoc manner, emphasizing the need for further improvements in the way that work processes and communications are defined if infrastructure organizations intend to deploy advanced information systems. The proposed IM-PMM would help the transaction development personnel (including transaction analysts, transaction designers, software developers, process modellers, and industry experts) to assess and benchmark the maturity of the work processes and communications in the domain of infrastructure. As part of the evaluation, the proposed IM-PMM is verified through testing and applying it in the domain of infrastructure management; future work will conduct validation through industry expert review.
\end{abstract}

Keywords: infrastructure management-process maturity model, maturity stage, process formalism, communication, transaction, infrastructure management, asset inventory reporting, asset condition assessment, benchmark

\section{Introduction}

Society's quality of life and sustainability depends heavily on the quality and sustainability of the public infrastructure, from roads and bridges to electrical grids and water systems, from hospitals and sport facilities to parks and public transit. Managing civil infrastructure systems is a complex task made more challenging as sustainability concerns introduce more stakeholder perspectives, wider-ranging objectives, and longer time-horizons. The authors are interested in supporting better infrastructure management practices through improved information systems, focusing on communications and information exchange rather than on specific 
computer applications. Examples of information exchange between infrastructure organizations or between infrastructure management systems include communications during disaster response (e.g. Is power available in this area? Who is responsible for this section of roadway? When will water be restored to this area?); coordination between buried utility agencies to develop a "call-before-you-dig" call center for excavations; or aggregating data from multiple infrastructure management software for the purpose of performing sustainability analysis or reporting to meet public sector accounting requirements.

As information systems mature in a typical organization, communications follow a general trend away from informal human-to-human communications towards computer-to-computer information exchange. In order to efficiently implement computer-to-computer information exchanges, the communications (referred to as transactions) between information system must be formally described, harmonized, documented, validated, and standardized for the industry. To formalize communications for computer-based exchange of information involves three issues: "how to formalize?" "what to formalize?" and "why to formalize". Elsewhere, the authors are exploring the first issue through the development of an ontology-supported transaction formalism protocol that the transaction development personnel would use to formalize transactions in the domain of infrastructure management effectively and efficiently. The second issue is addressed through identifying transactions in the domain of infrastructure management that have the greatest potential for IT improvement using a survey based approach. The third issue is dealt with exploring and examining the level to which these communications or transactions are formalized in the domain of infrastructure management using a maturity model technique, which is the core focus of this paper.

The authors have adopted the maturity model (MM) approach as a potential indicator of the level of work process and communication formalization. The maturity models are used to quantify and compare management practices for the purpose of benchmarking, determining strengths and areas for improvement, and identifying best practices (Harpham, 2006). They have been in use since 1970 (Gibson \& Nolan, 1974) and hundreds of MMs have been developed for information systems (Mettler \& Rohner, 2009; Becker \& Knackstedt, 2009) and other fields (Curtis \& Alden, 2007). There are relatively few in the domain of construction. All of the maturity models developed to date focus on the maturity of the way that organizations and processes are operated and managed. The primary interest of this research work is not on the operation and management of work processes but in the process design aspect of the work process and communications, which means the authors more focus on if and how processes and communications are formalized and defined and less on how these processes and communications are operated and managed. None of the available maturity models fulfill this requirement completely and; therefore, the authors were required to develop an assessment framework with slightly different definitions of the maturity levels. The assessment framework is named as Infrastructure Management-Process Maturity Model (IM-PMM).

This paper describes the development and application of the IM-PMM to assess the level to which work processes and communications are defined in current municipal infrastructure management. The terms "communication process(es)" "communication(s)" and "transaction(s)" are used interchangeably and are defined as any exchange of information between a sender and a receiver through a single or sequenced collection of messages. The paper first reviews techniques for benchmarking processes in Section 2. Section 3 briefly describes the development methodology and section 4 compares existing maturity models. Section 5 explains the development of the proposed IM-PMM. Section 6 describes the application of the assessment framework in a benchmarking survey. Section 7 analyzes the results, and section 8 describes the conclusions.

\section{Related Work in Process Maturity Models}

A central component of this research is to develop a technique for assessing the degree to which work processes and communications are formally defined in the domain of infrastructure management. The following section reviews a set of relevant applications of the maturity models in various related industries.

\subsection{Maturity Models Related to Project Management in the Construction Industry}

The Portfolio, Program, and Project Management Maturity Model, (P3M3) is a maturity model developed by the Office of Government Commerce (UK) and based on their Managing Successful Programs Methodology ${ }^{\mathrm{TM}}$ (MSP) standard. The P3M3 model can be described as a five-stage, discrete maturity model (OGC-APM, 2010). It is an advanced version of their Project Management Maturity Model. The focus of P3M3 is to provide effective guidance to organizations to establish process improvement initiatives at three different levels: portfolio, program, and project level.

The Organizational Project Management Maturity Model (OPM3) was developed by the Project Management Institute (PMI) and is based on the Project Management Body of Knowledge (PMBOK) standard (PMI, 2003). 
The focus of the OPM3 is to help organizations introduce best practices toward effective project management through understanding their project management processes, measuring the strengths and weaknesses of the processes, and suggesting improvements. It is also used to evaluate the organization's overall portfolio, program, and project processes.

The Berkeley's Project Management Process Maturity Model is a five-stage discrete model based on the PMBOK standard (Kwak \& Ibbs, 2000). The purpose of the model is to benchmark (measure and compare) project management practices and processes in diversified organizations, including AEC/FM organizations. This model uses a systematic and incremental approach to benchmark project management practices in organizations. In addition, this model incorporates the concept of continuous improvement at the project level to address advances in the project management knowledge.

The Interactive Capability Maturity Model (ICMM) was developed by the National Institute of Building Sciences (NIBS), based on the National Building Information Model Standards - NBIMS (McCuen \& Suermann, 2007). This model helps organizations evaluate their project management practices and processes in relation to Building Information Model (BIM) implementation.

The Indiana University BIM Proficiency Matrix (IU-BIM-PM) was developed by their University Architect's Office and is based on the IU BIM standards (IU, 2009). The focus of the Proficiency Matrix is to evaluate and benchmark the maturity of the project management practices and processes in organizations in line with the use of BIM technologies and help guide them towards improvement in future BIM implementations.

The Construction Supply Chain Maturity Model (CSC-MM) is a four-stage model based on the Fully Integrated and Automated Technology (FIATECH) Road Map (Vaidyanathan \& Howell, 2007). The CSC-MM framework helps construction supply-chain organizations to improve processes to achieve operational excellence.

The general summary of the existing initiatives related to maturity models in the construction domain is that: most use a five-stage model, most developments are attempting to improve the management of engineering in the field, and the development of maturity models is still an active research field related to the management of construction engineering projects.

\subsection{Maturity Models Related to the Software Industry}

The Capability Maturity Model Integration (CMMI), Version 1.3 and the Federal Aviation Administration-Integrated Capability Maturity Model (FAA-iCMM) are five-stage discrete models developed by the Software Engineering Institute (SEI, 2010). The CMMI is a process-improvement approach that helps organizations in the software industry to improve their processes through setting process improvement goals, providing guidance on how to achieve quality processes and benchmarking current processes. According to Ibrahim et al. (2001), the FAA-iCMM, Ver. 2 framework guides organizations to improve their ongoing processes using best practices.

\subsection{Maturity Models Related to Product Development in the Manufacturing Industry and Business Development}

The Dooley's New Product Development Maturity Model (Dooley et al., 2001) is used to benchmark the capabilities of processes and determine the impact of maturity on project performance. This model was developed based on a review of 39 new product development program maturity models related to business management.

The Object Management Group, a US based not-for-profit computer industry consortium, has developed the Business Process Maturity Model-BPMM (OMG, 2008). The BPMM's objective is to provide a technique for organizations to assess the maturity of processes, identify organizational strengths and weaknesses, and guide organizations to improve processes.

The Standardized Process Improvement for Construction Enterprises (SPICE) model is a five-stage discrete maturity model developed based on the European Foundation for Quality Management/Business Quality Foundation-Business Excellence Model (EFQM/BQM) standard (Hutchinson \& Finnemore, 1999). The SPICE is a framework that helps the construction industry to evaluate processes, identify strengths and weaknesses of the organization, and suggest and prioritize business development process improvements.

\section{Methodology to Develop and Apply the Infrastructure Management Process Maturity Model}

A five step methodology is devised to develop and apply the proposed IM-PMM. The proposed methodology is a modified version of the procedure developed by Becker and Knackstedt (2009) for developing maturity models. The five steps are as follows: (i) Define the problem - the problem was first explicitly defined in terms of the needs assessment to develop a new IM-PMM. (ii) Compare existing maturity models - existing maturity models 
were compared to assess gaps explicitly and to develop strategies for the development of the proposed IM-PMM. (iii) Develop the IM-PMM - the proposed IM-PMM was developed based on the review of the existing maturity models and the strategies developed in step 2. (iv) Apply the IM-PMM - the IM-PMM put into practice and applied in the domain of infrastructure management to test its' applicability. (v) Evaluate the IM-PMM - this step relates to evaluating the proposed IM-PMM. A brief description of step two, three, and four follows whereas step one is explained in the introduction section and step five (evaluation) is beyond the scope of this paper.

\section{Comparison and Summary of Maturity Models}

Table 1 compares the maturity models described above by modifying a table previously presented by Khoshgoftar and Osman (2009). The columns of the original table are expanded to include several additional maturity models (indicated by an asterisk "“*)) and the rows are modified from the original table by dropping several comparison criteria (rows in Table 1) that are not considered to be relevant in the context of this paper, while additional comparison criteria (indicated by a double asterisk “**”) are added to assess the maturity of work processes and communications from the perspective of the authors' research. The table also shows the comparison with the IM-PMM proposed by the authors and described in the following sections.

Table 1. A comparison of maturity models

\begin{tabular}{|c|c|c|c|c|c|c|c|c|c|c|c|c|c|}
\hline & \multirow[b]{3}{*}{ Comparis on Criteria } & \multicolumn{12}{|c|}{ Maturity Models } \\
\hline & & P3M3 & OPM3 & Berkley & I-CMM* & $\begin{array}{l}\text { IU-BIM- } \\
\text { PM* }\end{array}$ & $\mathrm{CSC}^{*}$ & СМMI & $\begin{array}{l}\text { FAA- } \\
\text { iCMM }\end{array}$ & Dooley* & ВРММ & SPICE* & $\begin{array}{l}\text { IM- } \\
\text { PMM* }\end{array}$ \\
\hline & & 1 & 2 & 3 & 4 & 5 & 6 & 7 & 8 & 9 & 10 & 11 & 12 \\
\hline i. & Author/Publisher & OGC & PMI & $\begin{array}{c}\text { Kawk } \\
\& \\
\text { Ibbs }\end{array}$ & NIBS & UoI & $\begin{array}{c}\text { VK } \\
\& \\
\text { HG }\end{array}$ & SEI & SEI & $\begin{array}{c}\text { Dooley } \\
\& \\
\text { Anderson }\end{array}$ & OMG & $\begin{array}{l}\text { Hutchinson } \\
\& \\
\text { Finnemore }\end{array}$ & $\begin{array}{l}\text { Zeb } \\
\text { et al. }\end{array}$ \\
\hline ii. & Domain & PM & $\mathrm{PM}$ & PM & PM & PM & $\begin{array}{l}\text { Supply } \\
\text { Chain }\end{array}$ & Software & Software & $\begin{array}{c}\text { Product } \\
\text { Development }\end{array}$ & Business & Business & Business \\
\hline iii. & $\begin{array}{l}\text { Growth/Maturity } \\
\text { Levels }\end{array}$ & 1 to 5 & ---- & 1 to 5 & --- & --- & 1 to 4 & 1 to 5 & 1 to 5 & --- & 1 to 5 & 1 to 5 & 1 to 5 \\
\hline iv. & $\begin{array}{l}\text { Discrete or } \\
\text { Continuous }\end{array}$ & Discrete & Continuous & Discrete & Continuous & Continuous & Discrete & Discrete & Discrete & Continuous & Discrete & Discrete & Discrete \\
\hline v. & Level of Details & High & $\begin{array}{c}\text { Extremely } \\
\text { High }\end{array}$ & High & Medium & Medium & Medium & High & High & Medium & High & Medium & Medium \\
\hline vi. & Refer to Standard & MSP & PMBOK & PMBOK & NBIMS & IUBIMS & FIATECH & --- & --- & --- & --- & $\mathrm{EFQM} / \mathrm{BQM}$ & IDM/VISI \\
\hline vii. & Maturity Definition & High & High & Medium & Medium & Medium & Medium & High & Medium & High & High & Medium & Medium \\
\hline viii. & $\begin{array}{l}\text { Evaluate Process } \\
\text { Maturity in terms of } \\
\text { Process Definition/** }\end{array}$ & Yes & Yes & Yes & Yes & Yes & Yes & Yes & Yes & No & Yes & Yes & Yes \\
\hline ix. & $\begin{array}{l}\text { Evaluate Actor-Role } \\
\text { Maturity in terms of } \\
\text { Actor-Role } \\
\text { Definition/** }\end{array}$ & No & No & No & No & No & No & No & No & No & No & No & Yes \\
\hline $\mathrm{x}$. & $\begin{array}{l}\text { Evaluate Information } \\
\text { Maturity in terms of } \\
\text { Information } \\
\text { Definition/** }\end{array}$ & No & No & No & Yes & No & No & No & No & No & No & No & Yes \\
\hline xi. & $\begin{array}{l}\text { Evaluate Message } \\
\text { Maturity in terms of } \\
\text { Message Definition/** }\end{array}$ & No & No & No & No & No & No & No & No & No & No & No & Yes \\
\hline xii. & $\begin{array}{l}\text { Applied to actual } \\
\text { Organizations }\end{array}$ & Yes & Yes & Yes & No & No & No & Yes & Yes & Yes & Yes & No & Yes \\
\hline xiii. & Assessment Difficulty & High & Low & Medium & High & High & Medium & High & High & Medium & High & Unknown & Low \\
\hline xiv. & Assesment Cost & High & Low & High & Medium & High & Medium & Medium & Medium & Medium & Medium & Unknown & Low \\
\hline $\mathrm{xv}$. & $\begin{array}{l}\text { Identify Weaknesses } \\
\text { and Strengths }\end{array}$ & Unknown & Yes & Yes & Yes & Yes & Yes & Yes & Yes & Yes & Yes & Yes & Yes \\
\hline xvi. & $\begin{array}{l}\text { Commitment for } \\
\text { Continuous } \\
\text { Assessment and } \\
\text { Improvement }\end{array}$ & Yes & Yes & Yes & No & No & Yes & Yes & Yes & No & Yes & Yes & Yes \\
\hline xvii. & $\begin{array}{l}\text { Results quantification } \\
\text { and Presentation }\end{array}$ & No & Yes & Yes & Yes & No & Yes & Unknown & Unknown & Yes & Unknown & No & Yes \\
\hline
\end{tabular}

In summary, maturity models assess the degree to which certain activities are managed in practice. A particular application of a maturity model assessment approach is characterized by two primary features: 
The assessment target - each maturity model is developed to assess a particular target: a specific domain, a type of process, or an explicit set of work processes and communications.

The maturity scale - maturity models assess their targets with respect to a prescribed maturity level scale that measures the degree of formalization, sophistication, or completeness of the management of the target. While each maturity model adapts and refines the discrete or continuous maturity levels to suit the specific context of their target, they typically progress through some form of the following stages of process formalization:

- The process is not being performed

- The process is performed in an ad hoc way (each actor develops their own approach)

- The process is formally defined, documented, and performed in a consistent way

- The process is actively managed, with process control, feedback, and continuous improvement

For this work, in assessing the formalization of work processes in the infrastructure management domain, the authors have adopted the same general approach used by all of the above models of assessing target processes against maturity levels. The maturity models investigated to date have focused on target domains such as project management and software development, based on standards such as PMBOK, MSP, IUBIM, and EFQM/BQM. None of these were found to be a good fit with the target domain of infrastructure management. For the IM-PMM; therefore, the authors have defined the target work processes and communications based on the infrastructure management framework of Vanier et al. (2009), as described in Section 5.1.

Furthermore, all of the maturity models investigated to date have focused on the maturity of the way that organizations and processes are managed. The authors' primary interest; however, is not in the operation and management of work processes themselves, but in the process design aspects of the work processes and communications. That is, the authors focus more on if and how the processes are formalized, and less on how the processes are then conducted and managed. As a result, the authors have developed slightly different definitions of the maturity levels (described in Section 5.2) than those used by the other maturity models discussed here.

Finally, although maturity model approaches typically assess the maturity level of the work process as a whole, the IM-PMM separates out certain important elements or features of the work process or communications in order to examine the process design in more detail. The elements that are examined for each work process and communication are process/transaction map definition, actor/role definition, information definition, and message definition (as explained in Section 5.3). Thus, while the proposed approach is similar in nature to other maturity models presented here, the requirements are sufficiently unique to warrant the development of a new IM-PMM.

\section{Development of the Infrastructure Management Process Maturity Model}

As described, the IM-PMM consists of three primary components: the work processes and communications that are to be evaluated, the maturity stages against which they are assessed, and the elements that are assessed for each work process or communication. Each of these is described in more detail in this section.

\subsection{The Work Processes and Communications Targets}

The targets of interest for this study are work processes and communications within the general domain of asset management. Vanier et al. (2009) define asset management as "a business process and decision-support framework that: (i) covers the extended service life of an asset; (ii) draws from engineering as well as economics; and (iii) considers a diverse range of assets". The specific domain of interest for this research work is the infrastructure management work function related to analyzing and selecting maintenance and rehabilitation alternatives as part of asset management planning for water, wastewater, and road infrastructure systems. This area of interest and these three specific infrastructure systems were selected because they are typically managed and controlled by only one body (municipal or local government) and they are rarely supported, investigated or researched in an integrated fashion; making it a suitable candidate for research. The importance of the municipal/local government segment of the construction industry is evident from the following facts:

- Municipal or local governments manage $20 \%$ of the Canadian civil infrastructure valued at $\$ 5.5$ trillion in 1999 constant dollars (Vanier \& Rahman, 2004), or approximately $\$ 1.1$ trillion of buildings, roads, bridges, water mains, sewers, etc.

- Approximately $90 \%$ of the Canadian population live in and served by municipalities.

- As public organizations, local governments have to ensure efficient utilization of the public money and; therefore, must be mature organizations. 
The infrastructure management work function is selected for three reasons: (i) regulations - imposition of new regulations that require municipal government to comply with public sector accounting board reporting requirements as part of the infrastructure management; (ii) identification - as part of an IT survey conducted in this research work, asset inventory and condition assessment reporting is identified in as one of the transactions that has the greatest potential for IT improvement; and (iii) rarely investigated domain - an area that is rarely researched and investigated. Drawing from the framework by Vanier et al. (2009), the following functions were taken as descriptions of the work processes to be assessed within the IM-PMM framework:

i. Asset inventory management: enumerating, listing and storing of the infrastructure asset information so that owners and managers of these assets are aware of what they own and where it is located.

ii. Asset condition assessment: evaluation of the existing condition of the assets so that owners and managers of assets are aware of the existing performance levels of the assets they own.

iii. Asset service life analysis: determination of the remaining or residual life of the assets based on the prevailing condition assessment of the asset.

iv. Asset life cycle cost analysis: is an assessment of the cost over the life cycle of the asset based on proposed maintenance scenarios.

v. Asset risk analysis: evaluation of the risks associated with the asset over its life cycle.

vi. Decision making analysis: analysis of proposed alternatives leading to the selection of the best alternative for maintenance or rehabilitation of the asset.

In addition to these work processes, the IM-PMM was used to evaluate different communications in the area of infrastructure management that were identified in the course of this research to be common to many of the municipalities in the survey. As explained earlier, communications (also referred to as transactions) are defined as any exchange of information between a sender and a receiver through a single or sequenced collection of messages. Several communications were identified in consultation with experts in the municipal infrastructure management, but only two general categories of communications were tested in this study:

i. Asset inventory reporting: reports identifying the existence, type, and location of infrastructure assets.

ii. Asset condition reporting: reports detailing the current condition of infrastructure assets.

\subsection{Maturity Stages}

The IM-PMM assesses the work processes and communications against five stages of maturity that reflect increasing levels of formalization or process maturity. The five stages for the proposed IM-PMM are selected due to: (i) compliance with existing maturity models - most of the existing maturity models are composed of five stages with each stage representing a maturity level in that specific application area; and (ii) formalization context - the focus of the proposed IM-PMM is on the design aspect of work processes and communications formalization in the domain of infrastructure management that is best assessed and represented through five levels of process design maturity. These stages selected for the proposed IM-PMM are discrete with medium level of details so that transaction development personnel can easily grasp and apply the content. The proposed stages follow general conventions for maturity models and are defined as follows:

Infancy Stage, (Process Formalism Unawareness) - In this stage, organizations carry out work processes or communications, but personnel are generally unaware of the idea that the work processes and communications can be formally defined, documented, and managed with respect to the four elements (process/transaction map, actor/role, information, and message definitions).

Preliminary Stage, (Process Formalism Awareness) - In the preliminary stage, personnel are aware of the idea of process formalism and know about the importance and definitions of the four elements, but these are not defined or documented in the process/communication in question. Each respondent carries out their role in the process as he/she sees fit, with little consideration of explicitly planning or documenting the process.

Reactive Stage, (Process Definition) - In the reactive stage, personnel carry out some explicit planning of the four elements in an ad hoc manner. The definitions are specific to a particular situation; they are dynamic and change frequently with time and context. The definitions are not documented for the purpose of future re-use.

Proactive Stage, (Process Standardization) - In the proactive stage, personnel define and document the four elements for future re-use, so that these work processes and communications are standardized over time. 
Integrated Stage, (Process Management) - In the integrated stage, processes are actively managed against the standardized process definitions. Data is collected to determine the success and effectiveness of the work process and communications, and on-going process/communication improvements are pursued.

\subsection{The Work Process and Communication Elements}

While other maturity model approaches typically assess the maturity level of the work process as a whole, the IM-PMM separates out certain important elements or features of the work processes or communications that can be assessed individually. According to the IDM (IAI-IDM, 2007) and VISI (VISI, 2007) standards, the design of work processes and communications centers around four important elements; including: (i) the design of a process/transaction map; (ii) the design of actors/roles; (iii) the design of the information content; and (iv) the design of messages. The design of a work process is characterized by the first three elements whereas the design of a communication is characterized by all the four elements. A work process or communication is said to be designed successfully if these elements are formalized properly. The four elements are examined as follows:

Process/Transaction Map Definition - The process/transaction map definition identifies and describes the activities to be completed and specifies the sequence or workflow logic linking the activities (IAI-IDM, 2007). In the case of communications, the comparable definition outlines the sequenced set of solo or atomic transactions in a bilateral or multilateral collaboration. It assesses the degree of formalization of these maps for work processes and communications.

Actor/Role Definition - The actor/role definition identifies actors, either individuals or organizations, who play certain roles in a given context. According to Zhang and El-Diraby (2009), actor role is defined as "a set of connected behaviors and attributes as conceptualized by actors in a given social position". The actions and responsibilities of actors with respect to work processes and communications depend upon the role that they are enacting. To formally describe work processes and communications, it is beneficial to define the actors and their roles in a given context. The actor/role definition assesses the degree of formalization of the respective role(s) that actors play in a given work process or communication (e.g. information sender or receiver roles).

Information Definition - This element assesses the degree to which the information inputs and outputs (i.e. to/from a work process, or the information content of a communication) are formally identified and described.

Message Definition - The message definition specifies the representational structure and format of the information (i.e. fully-structured, semi-structured and un-structured messages). While the information definition assesses the definition of what information is used in a process or communication (i.e., information content), it doesn't assess the definition of how the information is represented (i.e., information form). This may not be significant for work processes, but it is quite important for communications. Therefore, for communications only, the IM-PMM specifies the message definition to assess the level to which messages are formalized.

Figure 1 shows the overall assessment rubric for the proposed IM-PMM, with the different elements on the vertical axis - three elements for work processes plus a fourth element for communications, the maturity stages on the horizontal axis, and the corresponding interpretations in the intersecting cells. 


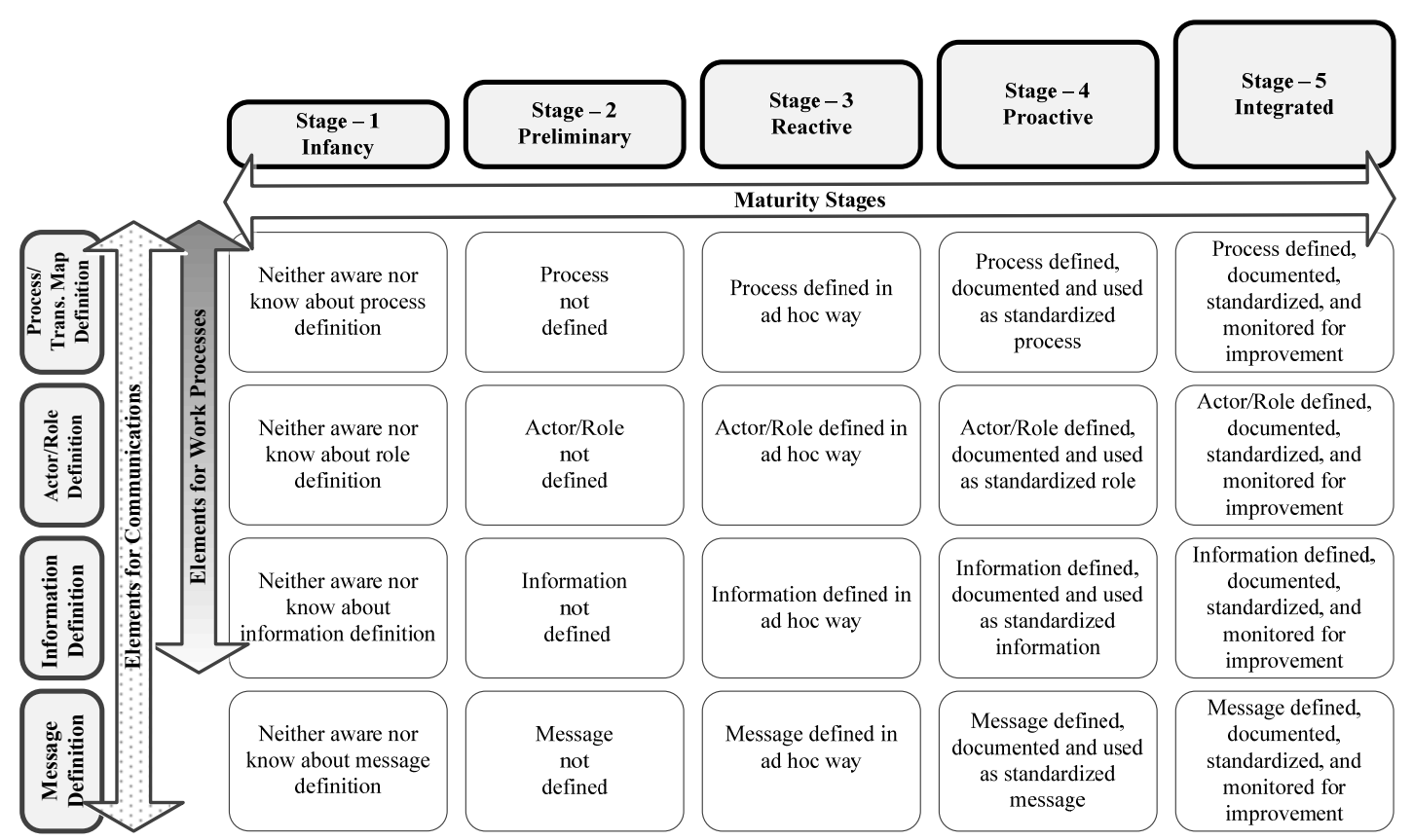

Figure 1. The infrastructure management-process maturity model (IM-PMM) rubric for assessing infrastructure work processes and communications

\section{Application of the Infrastructure Management Process Maturity Model}

Having developed the IM-PMM, the next step was to conduct a survey of selected municipalities to apply the model to the formalization practices for infrastructure management work processes and communications. The objective is to benchmark the level to which work processes and communications are formalized in infrastructure asset management practice, as well as to validate that the proposed IM-PMM is applicable.

\subsection{Planning and Design of the Survey}

Different types of survey methods were reviewed and a face-to-face interview method was selected due to "the flexibility to react to the respondent's situation, probe for more details, seek more reflective replies and ask questions which are complex or personally intrusive", (Glastonbury \& MacKean, 1991). The survey was developed by the first author as a structured questionnaire consisting of three parts: Part A focused on general information about the respondent, municipality, engineering department, and civil infrastructure systems; Part B benchmarked IT use by the municipality and captured information about the formalization of the municipality's infrastructure work processes and communications; and Part $\mathrm{C}$ was used to evaluate the protocol that was developed as part of the overall research. This protocol is a step-wise procedure that the municipalities can use to formalize work processes and communications in the domain of infrastructure management. The full questionnaire can be viewed at the research project website (IM-PMM, 2012).

To reduce the scope of the survey, it was decided to limit the survey to the water, wastewater and road infrastructure sectors of the municipal and regional governments. These three disciplines are good candidates for analysis as they are closely related and share considerable common data. The survey questionnaire was pre-tested in two stages by a small number of knowledgeable individuals. First, experts in the academic community reviewed the questionnaire (alpha testing) and identified shortcomings, which led to revisions. Second, in the beta testing stage, trial applications of the questionnaire were carried out with selected practitioners from the infrastructure industry. Their comments were evaluated, and alterations were incorporated as a second revision before the final execution of the questionnaire in the field.

\subsection{Target Population}

The target scope for the survey included 46 municipalities in southwestern British Columbia, Canada (within a practical and economic travelling distance for the researchers to carry out face-to-face interviews). Each municipality was first contacted with an invitation to participate in the survey. The number of municipalities contacted, along with their classification according to the Province of British Columbia, is as follows: 15 city municipalities (population greater than 5000), 8 town municipalities (populations between 2500 and 5000), 5 
village municipalities (population less than 2500) and 18 district municipalities (area greater than 800 hectares with population densities less than 5 people per hectare). Within these 46 municipalities, a total of 114 individuals were ultimately contacted personally in order to request permission to participate. Twelve (12) municipalities ( 8 cities and 4 districts) accepted the invitation to participate in the survey and agreed to face-to-face interviews (owing to multiple respondents at some municipalities there were a total of 20 individuals participating). Due to the low response rate from town and village municipalities, these two classifications of smaller municipalities were dropped from the analysis. Because of the small size, smaller population coverage, fewer human resources, and non-availability of the required expertise in the Town and Village municipalities, it was anticipated that their work processes and communications would be less defined and formalized than those of the city and district municipalities. To calculate the minimum sample size for each of the classifications, the authors assumed a confidence level of $95 \%$. Based on these parameters, a minimum sample size of five from each classification is sufficient to represent the target population.

\subsection{Conducting the Survey}

Once a confirmation to participate was received from a municipality, a consent form and a copy of the questionnaire were sent via e-mail to a participating municipality staff member, requesting respondents to complete Part A of the questionnaire on-line. This consisted of five questions about the municipalities and their civil infrastructure systems (total duration of 20 to 30 minutes). Expertise was self-assessed by the respondent in the water, wastewater and road sectors. The face-to-face interview followed, consisting of Part B (12 questions) and Part C (13 questions), for a total interview time of approximately 1 to 1.5 hours for each respondent.

\section{Analysis of Survey Results}

Figure 2 presents the survey results in three dimensions and Table 2 present the data in tabular form. The X-axis of Figure 2 represents the four elements, and then further decomposes each element into the five possible maturity levels. The identified work processes and communications are shown along the $\mathrm{Y}$-axis, while the Z-axis (vertical) identifies the number of responses received for each maturity level, providing the distribution of maturity level assessments for all elements. The work processes (six asset management sub-processes, described earlier) are represented in Figure 2 through alternate hatched cones whereas the communication processes are shown using hatched cylinders.

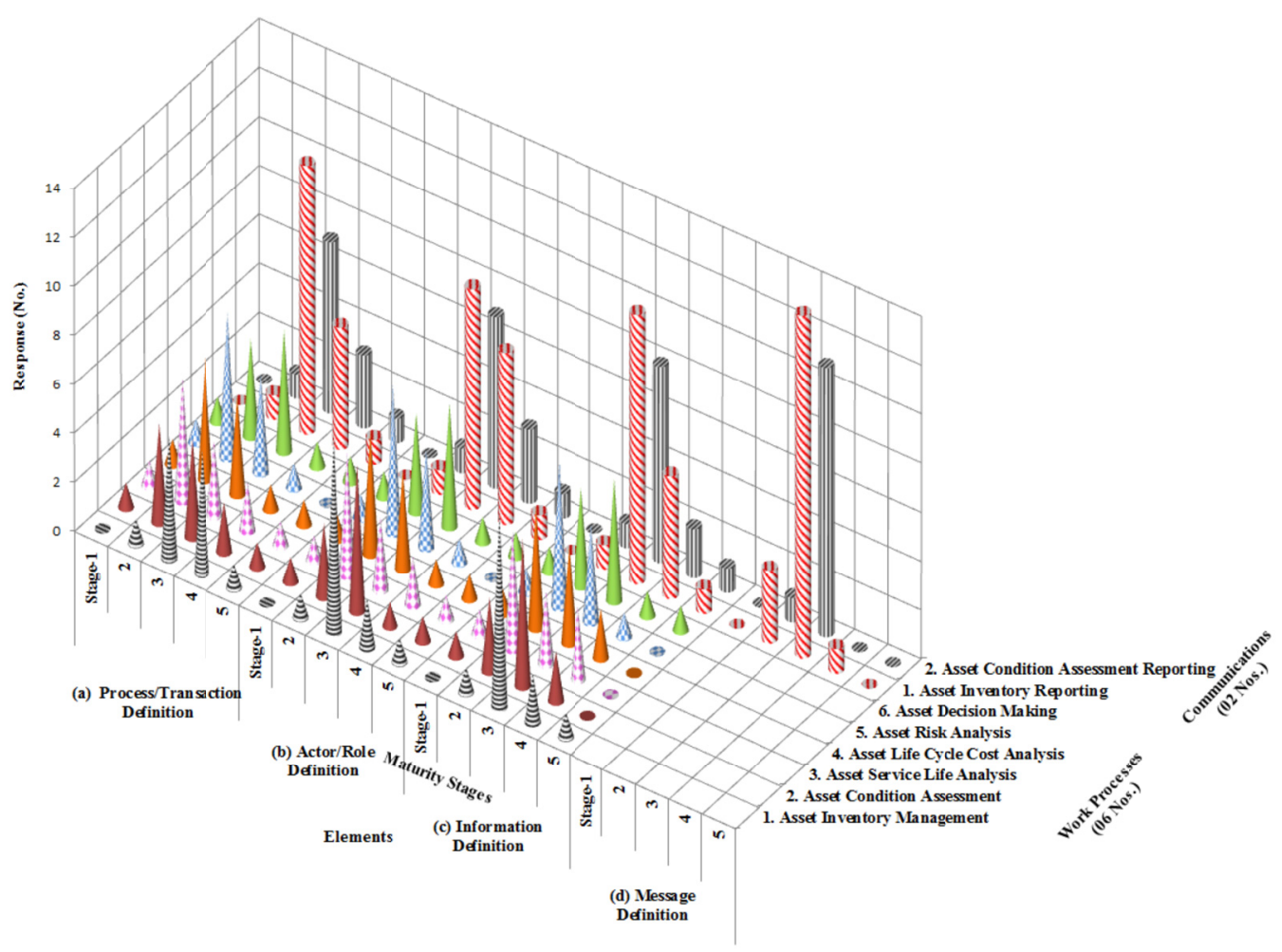

Figure 2. Benchmarking results showing the work processes and communications studied, the elements evaluated, and the number of responses found for each maturity stage 
Table 2 presents portions of the survey results in a tabular form. The columns represent the responses per stage in terms of the number of processes surveyed and the responses per element in terms of the sum of points scored.

Table 2. Work processes and communications - total responses per stage and responses per element

\begin{tabular}{|c|c|c|c|c|c|c|c|c|c|c|c|c|}
\hline \multirow[b]{2}{*}{$\begin{array}{l}\text { Work Processes and } \\
\text { Communications }\end{array}$} & \multicolumn{5}{|c|}{$\begin{array}{c}\text { Response/Stage } \\
\text { in terms of the Number of Processes }\end{array}$} & \multirow[b]{2}{*}{ Total } & \multicolumn{4}{|c|}{$\begin{array}{c}\text { Response/Element } \\
\text { in terms of Sum of Points Scored }\end{array}$} & \multirow[b]{2}{*}{ Average } & \multirow[b]{2}{*}{$\begin{array}{l}(+/-) \\
\text { Average }\end{array}$} \\
\hline & Stage-1 & Stage-2 & Stage-3 & Stage-4 & Stage-5 & & $\begin{array}{c}\text { Process/ } \\
\text { Transaction } \\
\text { Map }\end{array}$ & $\begin{array}{l}\text { Actor/ } \\
\text { Role }\end{array}$ & Information & Message & & \\
\hline \multicolumn{13}{|l|}{ Work Processes } \\
\hline Asset Inventory Management & 0 & 3 & 21 & 9 & 3 & 36 & 3.50 & 3.25 & 3.25 & & 3.33 & $21 \%$ \\
\hline Asset Condition Assessment & 3 & 10 & 16 & 5 & 2 & 36 & 2.81 & 2.80 & 2.76 & & 2.79 & $1 \%$ \\
\hline Asset Service Life Analysis & 3 & 15 & 9 & 7 & 2 & 36 & 2.75 & 2.75 & 2.67 & & 2.72 & $-1 \%$ \\
\hline Asset Life Cycle Analysis & 3 & 15 & 12 & 4 & 2 & 36 & 2.63 & 2.63 & 2.59 & & 2.62 & $-5 \%$ \\
\hline Asset Risk Analysis & 3 & 18 & 12 & 3 & 0 & 36 & 2.39 & 2.39 & 2.39 & & 2.39 & $-13 \%$ \\
\hline Asset Decision Making & 3 & 12 & 15 & 3 & 3 & 36 & 2.72 & 2.72 & 2.72 & & 2.72 & $-2 \%$ \\
\hline \multicolumn{13}{|l|}{ Communications } \\
\hline Asset Inventory Reporting & 0 & 6 & 45 & 18 & 3 & 72 & 3.37 & 3.48 & 3.37 & 2.92 & 3.29 & $1 \%$ \\
\hline Asset Cond. Assess. Reporting & 0 & 4 & 33 & 8 & 3 & 48 & 3.30 & 3.30 & 3.25 & 2.92 & 3.19 & $-1 \%$ \\
\hline Total (\%) and Avg. & $15(4 \%)$ & $83(25 \%)$ & $163(49 \%)$ & $57(17 \%)$ & $18(5 \%)$ & 336 & 2.93 & 2.92 & 2.88 & 2.92 & 2.91 & \\
\hline Total and Avg.-Work Processes & 15 & 73 & 85 & 31 & 12 & & 2.80 & 2.76 & 2.73 & & 2.76 & \\
\hline Total and Avg.-Communications & 0 & 10 & 78 & 26 & 6 & & $3.34^{\mathrm{a}}$ & $3.39^{\mathrm{b}}$ & $3.31^{\mathrm{c}}$ & 2.92 & 3.24 & \\
\hline Avg.-Communications (a, b, \& c) & & & & & & & & 3.35 & & & & $-13 \%$ \\
\hline
\end{tabular}

For the response per stage, responses were recorded for each process against each stage of the process maturity. For the response per element, responses were recorded for each process against each element of the proposed IM-PMM as a point scored. Each element was assigned a point value that was calculated as the product of the response and respective level. The last two columns show the average of the sum of points scored for each element and the percentage ( $+/$-) above or below the total average of 2.91 .

The top rows of the table show the six work processes and two communications. The bottom four rows show the totals in terms of the sum of responses per stage for all work processes and communications, with corresponding percentages and the average of the sum of points scored for each element. The survey results are analyzed, compared, and discussed as follows.

\subsection{Typical Maturity Levels}

The first observation that can be made about the results relates to the typical maturity levels identified from the survey. In general, relatively few respondents (4\%) assessed their municipality at maturity level 1 (i.e. infancy). One quarter (25\%) assessed their municipality at maturity level 2 (i.e. preliminary). The most common maturity level (49\%) was level 3 (i.e. reactive) where respondents follow ad hoc practices to define work processes and communications. At this level, respondents are aware of the four elements and use them to formalize the work processes and communications, but the process design is situation specific (ad hoc) and no process design documentation is maintained for future reuse. Few samples were found to be at higher maturity levels, with $17 \%$ at level 4 (i.e. proactive), where respondents make use of the elements to define the processes, standardize these processes and document them so that they can be reused in the future and $5 \%$ assessed their municipality at maturity level 5 (i.e. integrated), where work processes and communications are actively managed against the formal definitions and continuously monitored for improvement. This distribution gives an overall average maturity level of 2.91, suggesting that infrastructure practitioners are generally aware of and engaged in these processes and routinely define these processes, but that these process definitions are most commonly ad hoc, and are rarely being recorded, applied consistently, or actively managed against the four elements.

\subsection{Comparison of Different Work Processes and Different Communications}

The six aforementioned work processes all yielded similar levels of maturity for the first three elements $(2.80$, $2.76,2.73$ respectively) with an average level of 2.76 as shown in Table 2 . The only significant variations from this was that the inventory management process was $21 \%$ higher than the average of 2.76 for work processes with an average maturity level of 3.33 while the risk analysis process was $13 \%$ lower with an average maturity level of 2.39. Analysis of variance (ANOVA) analysis was performed to show that the differences between the different work processes were statistically significant (see Table 3, Test 1). 
Table 3. Results of tests for statistical significance using ANOVA technique

\begin{tabular}{|c|c|c|c|c|c|c|}
\hline Test & Null Hypothesis & $\mathbf{F}$ & Fcrit & $\mathbf{P}$ & Alpha & Result \\
\hline 1 & The processes being analyzed have no significant difference & 87.9 & 3.32 & $6.19 \mathrm{E}-08$ & 0.05 & Null hypothesis rejected \\
\hline 2 & The communications being analyzed have no significant difference & 5.87 & 10.12 & 0.09 & 0.05 & Null hypothesis accepted \\
\hline 3 & The elements being analyzed have no significant difference & 2.24 & 4.1 & 0.15 & 0.05 & Null hypothesis accepted \\
\hline 4 & $\begin{array}{l}\text { The four communication elements being analyzed have no significant } \\
\text { difference }\end{array}$ & 31.7 & 9.27 & 0.008 & 0.05 & Null hypothesis rejected \\
\hline 5 & $\begin{array}{l}\text { The work processes maturity being analyzed in the city and district } \\
\text { municipalities have no significant difference }\end{array}$ & 462.25 & 4.17 & $8.84 \mathrm{E}-20$ & 0.05 & Null hypothesis rejected \\
\hline 6 & $\begin{array}{l}\text { The communications maturity being analyzed in the city and district } \\
\text { municipalities have no significant difference }\end{array}$ & 320.06 & 5.31 & $1.05 \mathrm{E}-11$ & 0.05 & Null hypothesis rejected \\
\hline
\end{tabular}

In these results, the authors see three possible contributing factors. First, these six processes are roughly sequentially dependent, in that the later processes require the input of the earlier ones, but not vice-versa. For example, inventory management can be achieved without performing any condition assessment, but condition assessment can only be carried out having some form of the asset inventory system. Therefore, it is to be expected that the first process inventory management is likely to be more developed than the others. Second, condition assessment and performance prediction are often contracted out to consultants, so municipalities are likely to have less direct involvement with these processes and thereby less communication requirements. Third, the recent Canadian legislation related to implementation of the Public Sector Accounting Board 3150 (PSAB, 2009) requires Canadian municipalities to report their capital infrastructure (Tangible Capital Assets) in a consistent manner on their annual financial statements; this has prompted municipalities to formalize their management of infrastructure inventory, estimation of the cost of assets and their calculation of remaining asset life. Municipalities started reporting their TCAs on their annual financial statement in 2009 and are striving to formalize their processes to comply with PSAB 3150 requirements, but are still encountering difficulties with supplying asset condition data (CNAM, 2011).

In addition to the PSAB 3150 requirement, asset inventory and condition assessment information is typically exchanged between different departments in a municipality, between the accounting section and others (e.g. Municipal council, public works, engineering), between two or more municipalities when they share civil infrastructure system(s), between the municipality and consultants when specific tasks are outsourced, and between the municipalities and provincial government, when the provincial government requires asset inventory information from the municipalities for financial planning. There are numerous opportunities for transactions.

As can be seen in Table 2, the process/transaction map of the Asset Inventory Management sub-process was assessed as the highest of all samples; with an average maturity level of 3.50 (Figure 2 shows that approximately half of all samples are assessed at levels 4 or 5). These averages for the work processes are consistent with previous municipal infrastructure surveys (Vanier \& Rahman, 2004). These numbers suggest that higher levels of formalization are possible where there are sufficient drivers, such as PSAB 3150. In contrast, the asset risk analysis sub-process may be seen as a slightly less formalized process because municipalities mostly contract out project design and execution to consulting organizations that perform a risk analysis as part of the overall project. In addition, the risk analysis process is complex, requiring specialized software and expertise to perform it.

The ANOVA analysis confirmed that there was no significant difference found between the maturity levels of the two different types of communications: asset inventory reporting and asset condition assessment reporting (see Table 3, Test 2).

\subsection{Comparison of Different Elements}

Different elements on the X-Axis in Figure 2 were also compared with work processes and communications separately (see Table 3, Test 3); it was found that no statistically significant variations were observed between the overall maturity levels of the various elements assessed for the infrastructure processes.

For infrastructure communications; however, statistically significant differences were found between different elements (see Table 3, Test 4). The first three elements were all similar with an average maturity level of 3.35, while message definition was $13 \%$ lower with an average maturity level of 2.92 , indicating that the specification 
for message formats is not as widespread as the other elements. This may reflect the fact that the message definitions are currently intended more for communications that will be carried out by humans rather than computer-to-computer.

\subsection{Work Process Versus Communications}

The average maturity levels for communications in Table 2 were found to be a little higher than for work processes (3.24 versus 2.76). This is probably not a significant finding since all organizations were asked about the same work processes, while organizations were invited to self-report the maturity level of their own communications. This self-reporting on communications would likely skew the results towards those transactions that the organizations had more formally defined and would omit transactions for which the respondents were not aware.

\subsection{Comparison of Cities Versus Districts}

Statistically significant differences were found between the city and district municipalities for both work processes and communications maturity (see Table 3, Test 5 and 6 for the ANOVA techniques results). The average maturity levels of processes in the city and district municipalities were calculated to be 2.13 and 0.78 respectively (not shown in Table 2), which means processes are more formalized and defined in more populous cities in comparison to less densely populated district municipalities. The potential reasons for these differences include: availability of more financial and human resources to the city municipalities, availability of the technical skills required to model and design the processes, management vision and support to gain operational efficiencies through well-defined processes, and the use of the latest information technologies in the city municipalities (i.e. IT inherently requires more formalization of processes and communications).

\subsection{Overall Interpretation}

The overall interpretation of the survey results is that infrastructure organizations are routinely engaged in these infrastructure management processes, and that the notion of defining these work processes and communications is fairly common. However, the survey finds that these process definitions are currently very ad hoc, and there is a minimal application of standardized process formalizations, or active process management based on the process definitions (e.g. collecting process control data and pursuing continuous process improvement). The fact that some processes showed higher levels of formalization and that some infrastructure organizations were assessed at higher maturity levels indicates that the higher levels of formalization are possible within industrial practice when suitable drivers exist such as: expertise of the staff to define processes, policies within the organizations to define and standardize work processes and communications, prevalent use IT requiring processes to be formalized, etc.

These findings are significant because they show that the industry does not currently practice the highest degree of process formalization that is required for these work processes and communications to be implemented in advanced computer-based systems. It appears that better awareness of best practices and better support tools in the industry and their successful implementation could lead to increased process formalization. The IM-PMM could be used in the future to identify any such changes.

The authors believe that higher levels of process formalization are required in order to support the development and implementation of advanced computer-based communication systems. Furthermore, although the authors have not collected evidence to support an assertion, they hypothesize that higher levels of process formalization would lead to better management practices and better outcomes for the civil infrastructure systems being studied.

\section{Summary and Conclusions}

To better serve society, infrastructure organizations should deliver their services in an efficient and effective way. The potential exists to improve the management of civil infrastructure systems using advanced information systems. As information systems continue to evolve towards integrated automation, work processes and communications must increasingly be defined and formalized, thus transforming prevailing informal practices of information exchange between humans to more formal and well-defined work processes and communications.

In order to benchmark work processes and communications in the domain of infrastructure management, an IM-PMM was developed to assist organizations to position themselves on a process maturity continuum. The IM-PMM developed as part of this research work has five maturity stages along three core elements (namely, process/transaction map definition, actor/role definition, and information definition) to benchmark six asset management work processes, plus one additional element (i.e. message definition) to benchmark two asset management communication processes. Benchmarking was carried out using face-to-face interviews with experts from different city and district municipalities. 
From the analysis of the results, it is concluded that the items that are earlier in the general sequence of work processes (i.e. asset inventory management and condition assessment) are relatively more defined and formalized in comparison to later and more complex work processes like risk analysis and decision making analysis. No statistically significant variations were observed between the overall maturity levels of the various elements assessed for the work processes but the authors found a significant difference between the different elements in communications. Moreover, the average maturity levels for communications were found to be slightly higher than for work processes. Also, significant differences in maturity were found between the city and district municipalities for both work processes and communications.

In summary, the survey identified that work processes and communications are performed in an ad hoc way in the domain of infrastructure management. It is the view of the authors that the work processes and communications need to be better defined and formalized in order to help implement advanced information systems that, in turn, will help manage civil infrastructure systems efficiently.

The contribution of this research to theory is the development of the proposed IM-PMM whereas the contribution to practice is its' application in the domain of infrastructure management to assess the level to which work processes and communications are formalized. The proposed IM-PMM would enable the transaction development personnel to benchmark their work processes and communications in the domain of infrastructure management from the process design aspects, which means how processes and communications are formalized and designed and not on how these are conducted and managed. The limitations of the study in relation to generalizeability is the limited application of the proposed IM-PMM industry-wide, country-wide, department-wide, and process-wide. A policy framework is also needed to map out specific ways of driving the industry towards higher levels of process formalization in order to better manage civil infrastructure systems.

The authors strongly recommend that the proposed IM-PMM is to be applied in other domains to test for generalizeability. Industry-wide application - the proposed model needs to be applied in various industries to examine its' applicability in various industries including the AEC/FM industry. Country-wide application - the proposed MM should be applied in different municipalities across the country to broaden its' spectrum. Department-wide application - the IM-PMM should also be applied within different departments of the same municipality to test its' applicability. Process-wide - the proposed MM also needs to be applied to various processes within the same department.

A separate policy framework needs to be developed for city municipalities and another for district municipalities since there are significant differences found in the level of maturity between them. The strategies and policy recommendations contained in the respective frameworks should be able to address how with the higher levels of process formalization they can: (a) support the development and implementation of advanced computer-based communication systems; (b) lead to better management practices; and (c) lead to better outcomes for the civil infrastructure systems. The proposed research needs to be validated in future through industry experts as part of the evaluation process.

\section{Acknowledgements}

The authors gratefully acknowledge the Canadian Natural Sciences and Engineering Research Council (NSERC) for their support to this research.

\section{References}

Becker, J., \& Knackstedt, R. (2009). Developing maturity models for IT management - A procedure model and its application. Business and Information Systems Engineering, BISE - Research Paper, 213-222. http://dx.doi.org/ 10.1007/s12599-009-0044-5

CNAM. (2011). TCA best practices benchmarking survey findings report. Canadian Network of Asset Managers, TCA Program Office, The City of Calgary. Retrieved September 05, 2013 from http://www.cnam.ca/uploads/File/Association\%202011/TCA_Best_Practices_Benchmarking_Survey_Resul ts_Nov_2011.pdf

Curtis, B., \& Alden, J. (2007). The business process maturity model (BPMM): what, why and how. Business Process Modeling and Organizational Maturity, Capability Measurement, Continuous Visibility Delivered, $A$ Business Trend Column. Retrieved September 5, 2013, from http://www.bptrends.com/publicationfiles/02-07-COL-BPMMWhatWhyHow-CurtisAlden-Final.pdf

Dooley, K., Subra, A., \& Anderson, J. (2001). Maturity and its' impact on new product development project performance. Res Eng. Design 13, Springer-Verlag 2001, 23-29. 
Gibson, C. F., \& Nolan, R. L. (1974). Managing the four stages of EDP growth. Harvard Business Review 52, 76-88.

Glastonbury, B., \& MacKean, J. (1991). Handbook for research student in the social sciences. Chapter 19-Survey Methods, Vol. 1, Part 3, The Falmer Press, Taylor and Francis Inc., Bristol, 228.

Harpham, A. (2006). The APM group's assessment model for portfolio, program and project management, its PRINCE2 maturity model and their benefits to organizations. Retrieved September 5, 2013, from http://www.apmgroup.co.uk/nmsruntime/ saveasdialog.asp?

Hutchinson, A., \& Finnemore, M. (1999). Standardized process improvement for construction. Total Quality Management, 10, 4-5, 576-583. http://dx.doi.org/10.1080/0954412997550

IAI-IDM. (2007). Information delivery manual. International Alliance for Interoperability, building SMART Norwegian Chapter. Retrieved September 05, 2013, from http://www.buildingsmart.org/standards/idm

Ibrahim et al. (2001). The federal aviation administration - integrated capability maturity model, (FAA-iCMM). Version 2.0, An integrated Capability Maturity Model for Enterprise-wide Improvement, Published by the Federal Aviation Administration, USA.

IM-PMM. (2012). Questionnaire and glossary of items. Survey on the Use of Information Technologies in Municipal Infrastructure Management. Deptt. of Civil Eng. The University of British Columbia. Retrieved September 5, 2013, from http://137.82.46.184/projects/municipalitsurvey/Quest.pdf

IU. (2009). Indiana university building information modeling proficiency matrix - IU-BIM-PM. Indiana University Architect's Office. Retrieved September 5, 2013, from http://www.iu.edu/ vpcpf/consultant-contractor/standards/bim-standards.shtml

Khoshgoftar, M., \& Osman, M. (2009). Comparison of maturity models. 2nd IEEE International Conference on Computer Science and Information Technology, 297-301.

Kwak, Y. H., \& Ibbs, C. W. (2000). The Berkley project management process maturity model: Measuring the value of project management. Engineering Management Society, Proceedings of the IEEE, 1-5.

McCuen, T., \& Suermann, M. P. (2007). The interactive capability maturity model. AECbytes Viewpoint Sponsored by Bentley, Analysis, Research, and Reviews of AEC Technology, Viewpoint Technology, Viewpoint \# 33. Retrieved September 5, 2013, from http://www.aecbytes.com/viewpoint/2007/issue_33.html

Mettler, T., \& Rohner, P. (2009). Situational maturity models as instrumental artifacts for organizational design. Proceedings of the $4^{\text {th }}$ International Conference on Design Science Research in Information Systems and Technology (DESRIST), Malven, PA, USA. http://dx.doi.org/10.1145/1555619.1555649

OGC-APM. (2010). Portfolio, program, and project management maturity model - P3M3. An OGC website managed and published by the APM group in conjunction with OGC \& TSO, UK. Retrieved September 5, 2013, from http:/www.p3m3-officialsite.com/P3M3Model/P3M3Model.asp

OMG. (2008). Business process maturity model - BPMM. Version 1, Object Management Group, (OMG). Retrieved September 5, 2013, from http://www.omg.org/spec/BPMM/1.0/PDF

PMI. (2003). Organizational project management maturity model - OPM3. Project Management Institute, Newtown Square, Pennsylvania, PA, USA. Retrieved Sep 5, 2013, from http://marketplace.pmi.org/Pages/ProductDetail.aspx?GMProduct=00101463501

PSAB. (2009). Guide to accounting and reporting of tangible capital assets. Public Sector Accounting Board (PSAB), Canadian Institute of Chartered Accountants (CICA), Toronto, Ontario, Canada.

SEI. (2010). Capability maturity model integration - CMMI. Version 1.3, Software Engineering Institute, Carnegie Mellon. Retrieved September 5, 2013, from http://www.sei.cmu.edu/cmmi/

Vaidyanathan, K., \& Howell, G. (2007). Construction supply chain maturity model - conceptual framework. 15th International Group for Lean Construction Conference (IGLC15), East Lansing, MI, USA, 170-180.

Vanier, D. J., Newton, L. A., \& Rahman, S. (2009). Implementation details to support a generalized framework for municipal infrastructure management municipal infrastructure investment planning. National Research Council Canada, NRC Client Report No. B-5123.14, Ottawa, Canada.

Vanier, D. J., \& Rahman, S. (2004). MIIP report: survey on municipal infrastructure assets. National Research Council Canada, NRC Client Report No. B-5123.2, Ottawa, Canada. 
VISI. (2007). Voorwaarden scheppen voor invoering standaardisatie. Dutch Communication Management Standard, Developed by CROW - VISI Organization. Retrieved September 5, 2013, from http://www.crow.nl/

Zeb, J., \& Froese, T. (2011). Design and management of transactions in the AEC/FM industry using an ontological approach. $3^{\text {rd }}$ International/9 $9^{\text {th }}$ Construction Specialty Conference, Ottawa, Canada.

Zhang, J., \& El-Diraby, T. E. (2009). SSWP: A social semantic web portal for effective communication in construction. J. of Computers, Academy Publisher, 4(4), 330-337.

\section{Copyrights}

Copyright for this article is retained by the author(s), with first publication rights granted to the journal.

This is an open-access article distributed under the terms and conditions of the Creative Commons Attribution license (http://creativecommons.org/licenses/by/3.0/). 